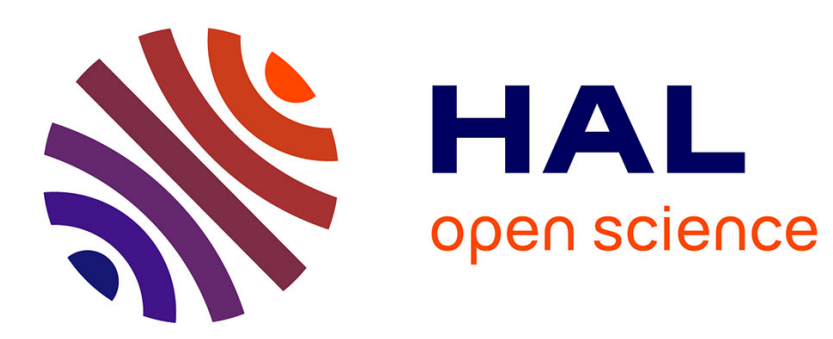

\title{
Microbeam dynamic shaping by closed-loop electrostatic actuation using modal control
}

Chady Kharrat, Eric Colinet, Alina Voda

\section{To cite this version:}

Chady Kharrat, Eric Colinet, Alina Voda. Microbeam dynamic shaping by closed-loop electrostatic actuation using modal control. PRIME 2007 - Microelectronics and Electronics Conference, 2007, Ph.D. Research in (PRIME 2007), Jul 2007, Bordeaux, France. pp.197 - 200. hal-00371315

\section{HAL Id: hal-00371315 https://hal.science/hal-00371315}

Submitted on 27 Mar 2009

HAL is a multi-disciplinary open access archive for the deposit and dissemination of scientific research documents, whether they are published or not. The documents may come from teaching and research institutions in France or abroad, or from public or private research centers.
L'archive ouverte pluridisciplinaire HAL, est destinée au dépôt et à la diffusion de documents scientifiques de niveau recherche, publiés ou non, émanant des établissements d'enseignement et de recherche français ou étrangers, des laboratoires publics ou privés. 


\title{
Microbeam dynamic shaping by closed-loop electrostatic actuation using modal control
}

\author{
Chady Kharrat ${ }^{1}$, Eric Colinet ${ }^{1}$ and Alina Voda ${ }^{2}$ \\ 1. CEA - LETI, MINATEC - Grenoble, France. \\ 2. GIPSA / LAG - Control System Department - Micro, nano sytems and SOC projects - Grenoble, France. \\ E-mail: chady.kharrat@cea.fr
}

\begin{abstract}
A closed-loop control approach for the dynamic shaping of a microbeam by electrostatic actuation is described. Starting from a desired displacements reference vector of $N$ small segments of the beam (representing the approximation of the continuous case), $n$ controllers ( $n$ is the number of considered modes) output the stresses that must be distributed throughout the beam, on the $N$ actuators. Because this reference may vary with time, the controllers are designed so that they accomplish good response dynamics, as well as performance and robustness specifications. The innovation in this method is that we control the dynamic coefficients associated to the modes of the microbeam and not directly the physical displacements in each small segment, which reduces the number of correctors from $N$ to the number of $n$ modes to control.
\end{abstract}

\section{INTRODUCTION}

The electrostatic actuation is a very common principle and occupies a very important place in MEMS field whether it is in AFM applications [1], mass sensors [2], data storage [3], micro-accelerometers [4], micromirrors [5], positioning for micromanufacturing [6] and many other applications. It involves simple circuitry and has a major advantage in the fact that it offers both electrostatic actuation as well as integrated detection, without the need for an additional position sensing device [7]. Its biggest disadvantage remains in the presence of nonlinear equations linking the input voltage to its resulting force as well as the ones linking the measured capacitance output to the displacement. Besides, the model of the microsystem's mechanical part describes nonlinear deformation behaviour due to nonlinear restoring forces and coupling between different modes of the structure [8]. In many applications, the system is considered as a second order mass-spring system, representing only the first mode by considering the beam as a plane one-block non-deformable mass moving or vibrating in one dimension, neglecting also the elongation of the beam as in [3-6]. This can be done when considering small displacements of the microstructure [9], which allows linearizing the model close to a working point [10].

Other applications which use deformable membranes describe the behaviour by two purely linear modes corresponding to whether bending stresses or tensile stresses are dominant [11]. In [12], the description of the microcantilever used as a sensor in AFM, includes different modes of vibrations at their specific resonant frequencies, based on the one-dimension Euler-Bernoulli equation.

This article aims to develop a closed-loop approach for the dynamic control of a deformable microbeam's shape using electrostatic actuation with two sets of electrodes from both sides of the beam. The approach is based on the detailed modelling of MEMS following its multiple modes and by taking into consideration the existing mechanical and actuation nonlinearities and couplings. Its application can be useful in micropumps as well as micromirrors. Some works already present in the literature contributed to the conception of the electrostatic MEMS especially for deformable micromirrors. Some of them are based on solving the non-linear mechanical inverse problem to calculate the ideal pressure distribution and then the voltages which reduce the residual deformations by quadratic programming problem [13]. Others use the deformation of the electrode shape to obtain a determined relation voltage / capacity on output [14] but are used only for steady state solutions. A nonlinear electrostatic control from the two sides of an oscillating cantilever is also described in [15] and serves as a reference for the electrostatic actuation.

Our work is divided into three parts:

First, we study the detailed modelling of the microbeam and the modal analysis of its behaviour. This is essential to understand the relation between the external forces and the deformation movement which vary according to the position throughout the beam. Having obtained a precise model, a control law can be designed to achieve the defined goals. In the second part, a mode-based-control method is studied to design a controller achieving desired specifications on the dynamics of each mode as well as good performances and robustness of the regulation. In our work, the controller used so far is a PID which has the advantages of being simple to integrate on the microscale device without requesting the implementation of complex electronic circuitry.

The third part shows the implementation of the complete device on Matlab using numerical parameters and exhibits the simulation results. The System's performance evaluation is done by applying disturbances on input as well as noise on output associated to electronic circuitry and ADC converters. 


\section{DEFORMATION MODELLING}

The behaviour of the deformation of a rectangular microbeam with length $l$, thickness $e$, width $h$, subjected to an external distributed strength obeys to the following differential equation:

$$
\begin{aligned}
E I \frac{\partial^{4} w(x, t)}{\partial x^{4}}+ & T(w(x, t)) \frac{\partial^{2} w(x, t)}{\partial x^{2}}+b \frac{\partial w(x, t)}{\partial t} \\
& +\rho S \frac{\partial^{2} w(x, t)}{\partial t^{2}}=f(x, t)
\end{aligned}
$$

With $S=$ h.e the transversal section of the beam, E the Young's modulus, $I$ the moment of inertia, $\rho$ the density, $b$ the friction coefficient associated to the interaction with the surrounding fluid, $w(x, t)$ the time dependent transverse displacement at position $x$, and $T(w)$ is the stress associated to the elongation of the beam.

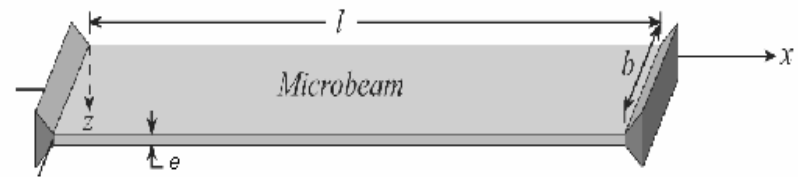

Figure 1. A clamped-clamped microbeam with length $l$, thickness $e$, and width $h$.

Following Galerkin procedure of standard modal analysis, $w(x, t)$ can be written as:

$$
w(x, t)=\sum_{k=1}^{n} a_{k}(t) \cdot w_{k}(x)
$$

where $w_{k}(x)$ are the $n$ mode shape vectors verifying the equation $\frac{\partial^{4} w_{k}(x)}{\partial x^{4}}=\lambda_{k}^{4} w_{k}(x), \quad \lambda_{k}^{4} \quad$ the correspondent eigenvalues and $a_{k}(t)$ the dynamic related coefficients.

A solution of this equation, with respect to the boundary conditions and considering a clamped-clamped microbeam (Fig. 1) is the space formed by the vectors:

$$
\begin{aligned}
w_{k}(x)= & A_{k} \varphi_{k}(x)=A_{k}\left[\cos \left(\lambda_{k} x\right)-\frac{\cos \left(\lambda_{k} l\right)-\cosh \left(\lambda_{k} l\right)}{\sin \left(\lambda_{k} l\right)-\sinh \left(\lambda_{k} l\right)} \sin \left(\lambda_{k} x\right)\right. \\
& \left.-\cosh \left(\lambda_{k} x\right)+\frac{\cos \left(\lambda_{k} l\right)-\cosh \left(\lambda_{k} l\right)}{\sin \left(\lambda_{k} l\right)-\sinh \left(\lambda_{k} l\right)} \sinh \left(\lambda_{k} x\right)\right]
\end{aligned}
$$

where $\lambda_{k}$ verify $\cos \left(\lambda_{k} l\right)=1 / \cosh \left(\lambda_{k} l\right)$ which leads to $\left(\lambda_{k} l\right)=\left[\begin{array}{llllll}4.73 & 7.85 & 10.99 & 14.13 & 17.27 & \cdots\end{array}\right]$.

Choosing $A_{k}=\left(\int_{0}^{l} \varphi_{k}^{2}(x) d x\right)^{-1 / 2}$ results in an orthonormal base of defined $w_{k}(x)$.

Using equation (2) in (1) and projecting on each vector $w_{i}$, we get $n$ equations representing the $n$ modes:

$$
E I a_{i} \lambda_{i}^{4}+T(w) \sum_{k=1}^{n} a_{k}\left\langle\frac{d^{2} w_{k}}{d x^{2}} \mid w_{i}\right\rangle+b \cdot \dot{a}_{i}+\rho \cdot S \cdot \ddot{a}_{i}=\left\langle f \mid w_{i}\right\rangle
$$

These $n$ equations can be written in a matrix form:

$$
K X+N(X)+B \dot{X}+M \ddot{X}=F
$$

where $X=\left[\begin{array}{llll}a_{1}(t) & a_{2}(t) & \cdots & a_{n}(t)\end{array}\right]^{T}, M=\rho \cdot S \cdot I_{n}$,

$$
K=E I\left[\begin{array}{cccc}
\lambda_{1}^{4} & 0 & \cdots & 0 \\
0 & \lambda_{2}^{4} & & \vdots \\
\vdots & & \ddots & 0 \\
0 & \cdots & 0 & \lambda_{n}^{4}
\end{array}\right], B=\left[\begin{array}{cccc}
b_{1} & 0 & \cdots & 0 \\
0 & b_{2} & & \vdots \\
\vdots & & \ddots & 0 \\
0 & \cdots & 0 & b_{n}
\end{array}\right] \text {, }
$$$$
F=\left[\begin{array}{c}
\left\langle f \mid w_{1}\right\rangle \\
\left\langle f \mid w_{2}\right\rangle \\
\vdots \\
\left\langle f \mid w_{n}\right\rangle
\end{array}\right], \text { and } N(X)=T(w) \cdot A \cdot X \text { with }
$$$$
A=\left[\begin{array}{cccc}
\left\langle\frac{d^{2} w_{1}}{d x^{2}} \mid w_{1}\right\rangle & \left\langle\frac{d^{2} w_{2}}{d x^{2}} \mid w_{1}\right\rangle & \cdots & \left\langle\frac{d^{2} w_{n}}{d x^{2}} \mid w_{1}\right\rangle \\
\left\langle\frac{d^{2} w_{1}}{d x^{2}} \mid w_{2}\right\rangle & \ddots & \vdots \\
\vdots & & \ddots & \vdots \\
\left\langle\frac{d^{2} w_{1}}{d x^{2}} \mid w_{n}\right\rangle & \cdots & \cdots & \left\langle\frac{d^{2} w_{n}}{d x^{2}} \mid w_{n}\right\rangle
\end{array}\right]
$$

The stress due to the elongation of the beam can be expressed by the equation $T(w)=E . S . \frac{\Delta l}{l}$, where $\Delta l=\frac{1}{2} \int_{0}^{l}\left(\frac{d w}{d x}\right)^{2} d x=\frac{1}{2} \int_{0}^{l} \sum_{k=1}^{N} \sum_{l=1}^{N} a_{k} \frac{d w_{k}}{d x} \frac{d w_{l}}{d x} a_{l} d x=-\frac{1}{2}\left(X^{T} . A . X\right)$ Thus, one has $N(X)=-\frac{E S}{2 l}\left(X^{T} \cdot A \cdot X\right) \cdot A \cdot X$.

\section{CONTROLlER DESIGN}

In this section, a controller is designed so that it ensures the tracking of the microbeam displacements set to a given dynamic reference. The force calculated by the controller is then transformed to the desired voltage properly distributed on $N$ electrodes (existing on both sides of the microbeam, exhibiting positive and negative forces). Increasing the number of the actuating electrodes leads to a better approximation of the continuous case allowing better approximated continuous deformation reference. In the same time it increases the complexity of the micromanufacturing, the number of control voltage sources and the coupling between the electrodes which we neglect for the moment, willing to take it into consideration in future works especially at the NEMS scale. Besides, we consider a direct accessibility to the $N$ displacement values. A rudimentary idea is to calculate the errors between the measured displacements and their references for each segment and then to produce the desired local force which must be applied to achieve tracking at a cost of using a huge network of $N$ controllers. Also, one will be dealing with the equation (1) directly and no desired dynamics can be directly imposed on the displacement response. A better idea is to control the dynamic coefficients associated to the eigenvectors reasoning in the eigenmodes space. Having all $a_{k}(t)=a_{k \text { ref }}(t)$ means $w(x, t)$ has the shape of $w_{r e f}(x, t)$.

Using this method, the number of PID controllers used is reduced to $n$ which is the number of modes taken into consideration. When $n$ is high, the shaping of the beam is more accurately controlled. In our work, we limit it to the $198^{\text {first five modes. }}$ 
Let $e(x, t)=w(x, t)-w_{\text {ref }}(x, t)$ be the vector of $N$ elements representing the errors of tracking in each segment of the beam. Projecting this vector on the eigenvectors base results in $n$ values of:

$$
e_{k}(t)=a_{k}(t)-a_{k r e f}(t)
$$

The transfer function relating $a_{k}(t)$ to $f_{k}(t)$ neglecting the nonlinear term is expressed by the following:

$$
G_{k}(s)=\frac{1}{m s^{2}+b_{k} s+k_{k}}
$$

$m, b_{k}$ and $k_{k}$ are the $k^{\text {th }}$ parameters of the matrices $M, B$ and $K$ described above.

For a determined desired dynamic response characterised by the damping coefficient $\xi_{d}$ and the natural pulsation $\omega_{d}$, the closed-loop desired transfer function for all the modes is written as:

$$
T_{k}(s)=\frac{\omega_{d}^{2}}{s^{2}+2 \xi_{d} \omega_{d} s+\omega_{d}^{2}}
$$

For this purpose, PID controllers with the transfer function:

$$
K_{k}(s)=P\left[1+\frac{1}{T_{i} s}+\frac{T_{d} s}{1+\frac{T_{d}}{N} s}\right]
$$

are chosen with the following parameters:

$$
\begin{array}{ll}
T_{i k}=b_{k} / k_{k}-\frac{1}{2 \xi_{d} \omega_{d}} & P_{k}=\frac{\omega_{d} T_{i k} k_{k}}{2 \xi_{d}} \\
T_{d k}=\frac{m / k_{k}}{T_{i k}}-\frac{1}{2 \xi_{d} \omega_{d}} & \frac{T_{d k}}{N_{k}}=\frac{1}{2 \xi_{d} \omega_{d}}
\end{array}
$$

The force calculated is then equal to:

$$
f_{k}(t)=P\left(e_{k}(t)+T_{d_{k}} \dot{e}_{k}(t)+\frac{\int e_{k}(t) d t}{T_{i_{k}}}\right)
$$

Thus we obtain a vector $F(t)$ of $n$ values. The distributed force is recomposed with the following operation:

$$
f(x, t)=\sum_{k=1}^{n} f_{k}(t) \cdot w_{k}(x)
$$

Knowing that the electrostatic force between the capacitor plates (the electrode and the segment of the beam) generated by applying a voltage $u$ is $f=\frac{1}{2} \frac{\varepsilon_{0} S \cdot u^{2}}{(g-w)^{2}}$, the needed voltage for each electrode is calculated by the following equation:

$$
v(x, t)=|g-w(x, t)| \cdot \sqrt{\frac{2|f(x, t)|}{\varepsilon_{0} S}}
$$

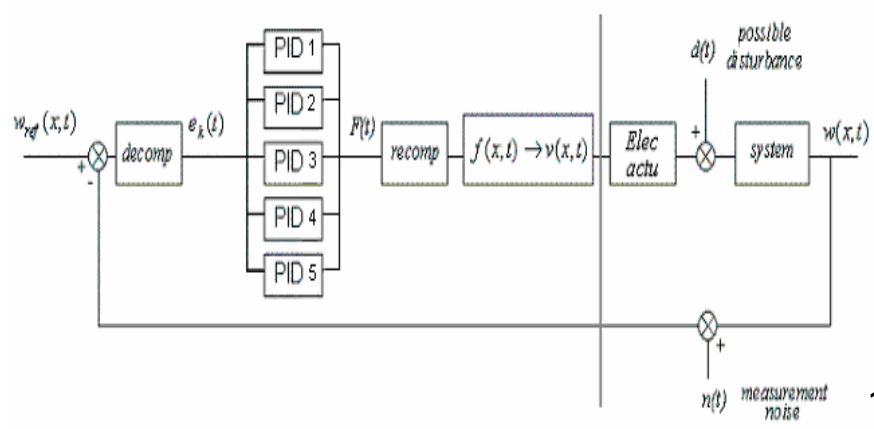

$w(x, t)$

Figure 2. Detailed diagram of the closed-loop system and is applied on the higher electrode if $f(x, t)>0$, and on the lower electrode if $f(x, t)<0$.

\section{SimULATIONS AND RESULTS}

The above microbeam model is implemented in Simulink/Matlab. The degrees of freedom for the mechanical part are the geometry of the beam, the number of modes taken into consideration and the physical parameters associated to these modes defining their quality factor.

A beam with length $l=13,35 \mu m$, width $h=0.2 \mu m$ and thickness $e=0.2 \mu \mathrm{m}$ is considered. The moment of inertia is then $I=h \cdot e^{3} / 12$. The gap between the electrodes and the surface of the beam is $g=0.1 \mu \mathrm{m}$, the density is $\rho=2.232 .10^{3}$, the young's modulus is $E=169.10^{9}$ and so we have $k_{k}=E . I . \lambda_{k}^{4}$ and $b_{k}=\frac{\sqrt{k_{k} \cdot m}}{Q}$, with $m=\rho . e . h$ and $Q=4000$ for all the modes.

The bigger $\omega_{d}$ is, the bigger is the system's bandwidth with higher response speed. Yet, the noise effect on the input is bigger, but with a better disturbance rejection. On the other hand, when the damping $\xi_{d}$ is bigger, we have less overshooting with slower response speed and less disturbance rejection, but the noise is more attenuated. We specified desired natural pulsation to be $\omega_{d}=5.10^{10} \mathrm{rad} / \mathrm{s}$ and $\xi_{d}=0.8$ allowing good conciliation between all specifications.

Simulation results are shown on fig. 3, 4, 5 and 6. Control voltage inputs $v_{k}(x, t)$, the reference shape and the output $w(x, t)$ are presented at a fixed time $t_{f}$ to show their distribution on the beam (fig. 3). The dynamic evolution of the beam's shape is finally represented in fig. 4. The reference displacements represented by the reference dynamic coefficients $a_{k r e f}(t)$ are shown in fig. 5 and the modal reference tracking is described by the modal dynamic errors (fig. 6).

In addition to the natural dynamic tracking errors which depend on the controller design, slight errors exist because of the nonlinear term $N(X)=-\frac{E S}{2 l}\left(X^{T} . A \cdot X\right) \cdot A \cdot X$ due to the elongation of the beam which is taken into consideration in simulations. Nevertheless, the residuals are less than $1 \%$ of their correspondent references.

Tests on closed-loop system's performance are done by adding disturbance $d(t)$ on the force input. Also the effect of detection noise $n(t)$ has been taken into consideration (power distribution equal to $1 e-42 F^{2} / H z$ on a bandwidth of $1 \mathrm{MHz}$ ). Satisfactory results are obtained; however, the design of an observer in the control loop would help displacement reconstruction, surmounting the noise effect. This point out one of our future interests.

\section{CONCUSION}

This paper details the design of a fully integrable 199 control loop for a microbeam dynamic shaping by 

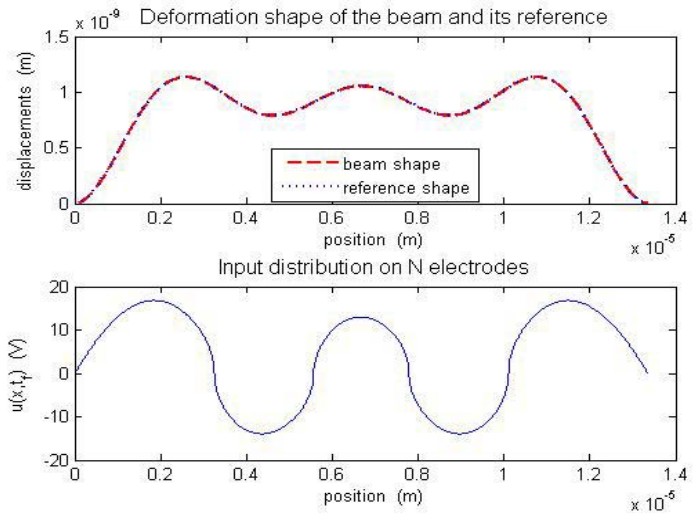

Figure 3. On top: reference shape of the microbeam and output shape on fixed time instant $t_{f}$. On bottom: corresponding voltage input distributed on the electrodes on the same time $t_{f}$.
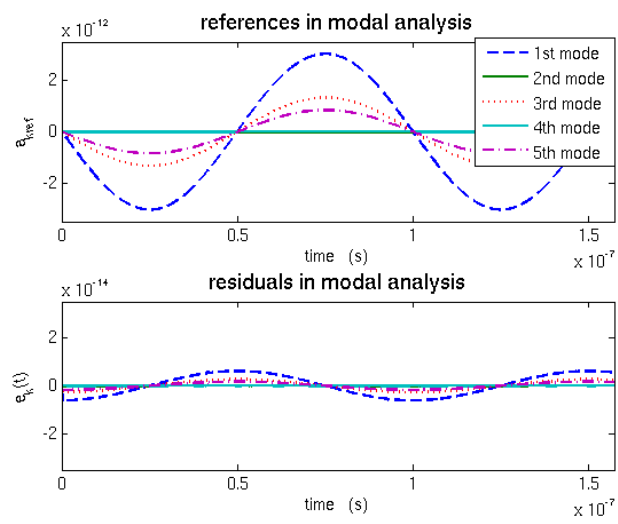

Figure 5. On top: Each curve represents the evolution of a reference dynamic coefficient of one of the 5 modes. On bottom: Tracking errors of the dynamic coefficients of each mode. For a sinusoidal time function reference, the errors do not exceed $1 \%$ of the reference values.

electrostatic actuation done with a set of $N$ small electrodes .on both sides of the microbeam. First of all, the detailed modelling of the structure using modal analysis is presented. The control of the beam's shape with dynamic references is accomplished by a set of $n$ PID controllers after projecting the tracking errors on the modal space of dimension $n$. This procedure was successfully simulated on Matlab and control specification with good performance and robustness were obtained.

\section{REFERENCES}

[1] Butt H.J., "Electrostatic interaction in atomic force microscopy", Biophys J., Vol. 60(4), pp. 777-785, 1991.

[2] Li Y.C., Ho M.H., Hung S.J., Chen M.H., Lu M., "CMOS micromachined capacitive cantilevers for mass sensing", Journal of Micromechanics and Microengineering, Vol. 16, Num. 12, pp. 26592665,2006

[3] Lu M.S., Feeder G.K. "Position control of parallel-plate microactuators for probe-based data storage", Journal of Microelectromechanical systems, Vol. 13, pp. 759-769, 2004.

[4] Soen J., Voda A., Condemine C., "Controller design for a closed-loop micromachined accelerometer", Control engineering practice 15,57 68, 2007.

[5] Yi Z., Francis T., Guangya Z., Fook S.C., "Fast and precise positioning of electrostatically actuated dual-axis micromirror by multi-loop digital control", Sensors and Actuators A: Physical, 2006.

[6] Xie H., Fedder G.K., "Vertical comb-finger capacitive actuation and sensing for CMOS-MEMS", Sensors and Actuators A, Vol. 95, pp. 212-221, 2002

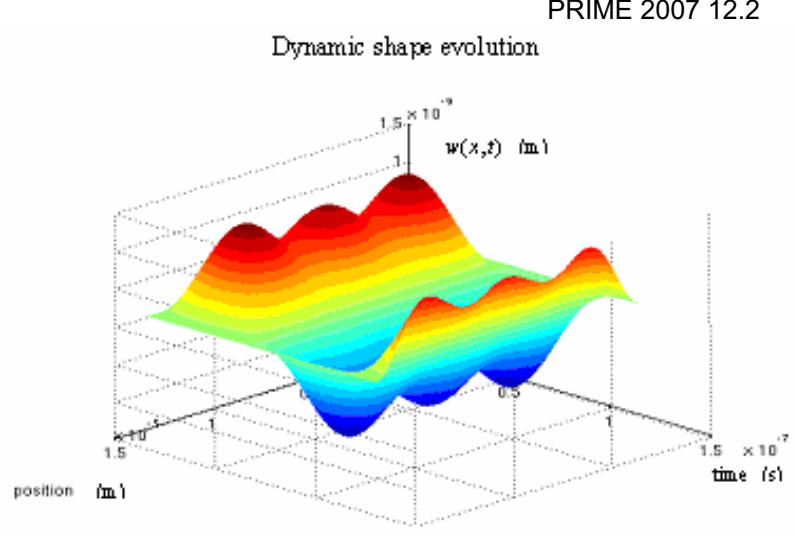

Figure 4. Dynamic evolution of the beam's shape in time, following a sinusoidal time varying reference.
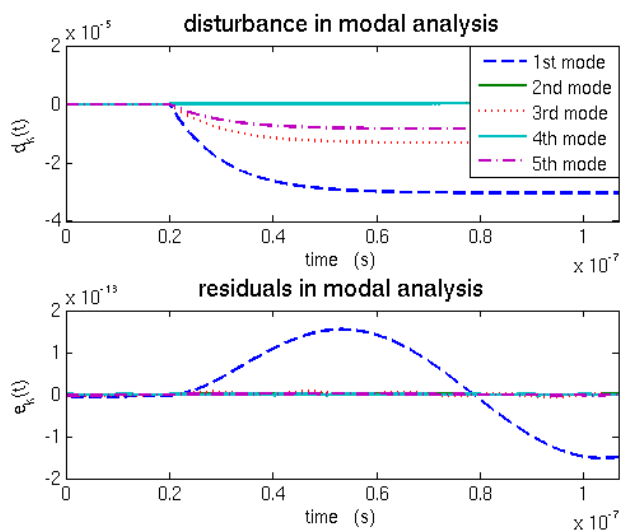

Figure 6. On top: Coefficients of the projected disturbance on the 5 modes. The disturbance is added on the voltage input. On bottom: Resulting tracking errors of the displacement dynamic coefficients of each mode.

[7] Napoli M., Bamieh B., Turner K., "A Capacitive microcantilever: Modelling, validation, and estimation using current meaurements", Journal of Dynamic Systems, Measurement, and Control, Vol. 126, pp. 319-326, 2004.

[8] Younis M., Abdel-Rahman E., Nayfeh A., "A Reduced-Order Model for Electrically Actuated Microbeam-Based MEMS", Journal of Microelectromechanical systems, Vol. 12, No. 5, pp. 672-680, 2003.

[9] Juillard J., Colinet E., "Modelling of nonlinear circular plates using modal analysis: simulation and model validation", J. Micromech. Microeng., Vol. 16, pp. 448-456, 2006.

[10] Abdel-Rahman E., Nayfeh A., "Secondary resonances of electrically actuated resonant microsensors", J. Micromech. Microeng., Vol. 13, pp. 491-501, 2003.

[11] Wang P.K.C, Hadaegh F.Y., "Computation of static shapes and voltages for micromachined deformable mirrors with nonlinear electrostatic actuators", IEEE J. Microelectromech. Syst., Vol. 5, pp. 205-219, 1996

[12] Hrouzek M., Besancon-Voda A., Stark M., Chevrier J., "Model of the cantilever used as a weak force sensor in Atomic Force Microscopy", 16th World Congress of IFAC, Prague, Tcheq Rep, 2005.

[13] Juillard J., Cristescu M., "An inverse approach to the design of adaptive micro-mirrors", J. Micromech. Microeng., Vol.14, pp. 347$355,2004$.

[14] Hung S., Senturia S., "Tunable capacitors with programmable capacitance-voltage characteristic", Technical Digest Solid-State Sensor and Actuator Workshop, pp. 292-295, 1998.

[15] Besancon G., Voda A., Colinet E., "Oscillation control in a vibrating cantilever nonlinear system", to appear in European Control Conference, ECC07, July, 2007. 